Document downloaded from:

http://hdl.handle.net/10251/93297

This paper must be cited as:

Alducin G.; Contero, M.; JORGE MARTIN GUTIÉRREZ; Guerra-Zubiaga D.; Johnson M.D. (2011). Productivity Improvement by Using Social-Annotations

about Design Intent in CAD Modelling Process. Lecture Notes in Computer Science. 6778:153-161. doi:10.1007/978-3-642-21796-8_16

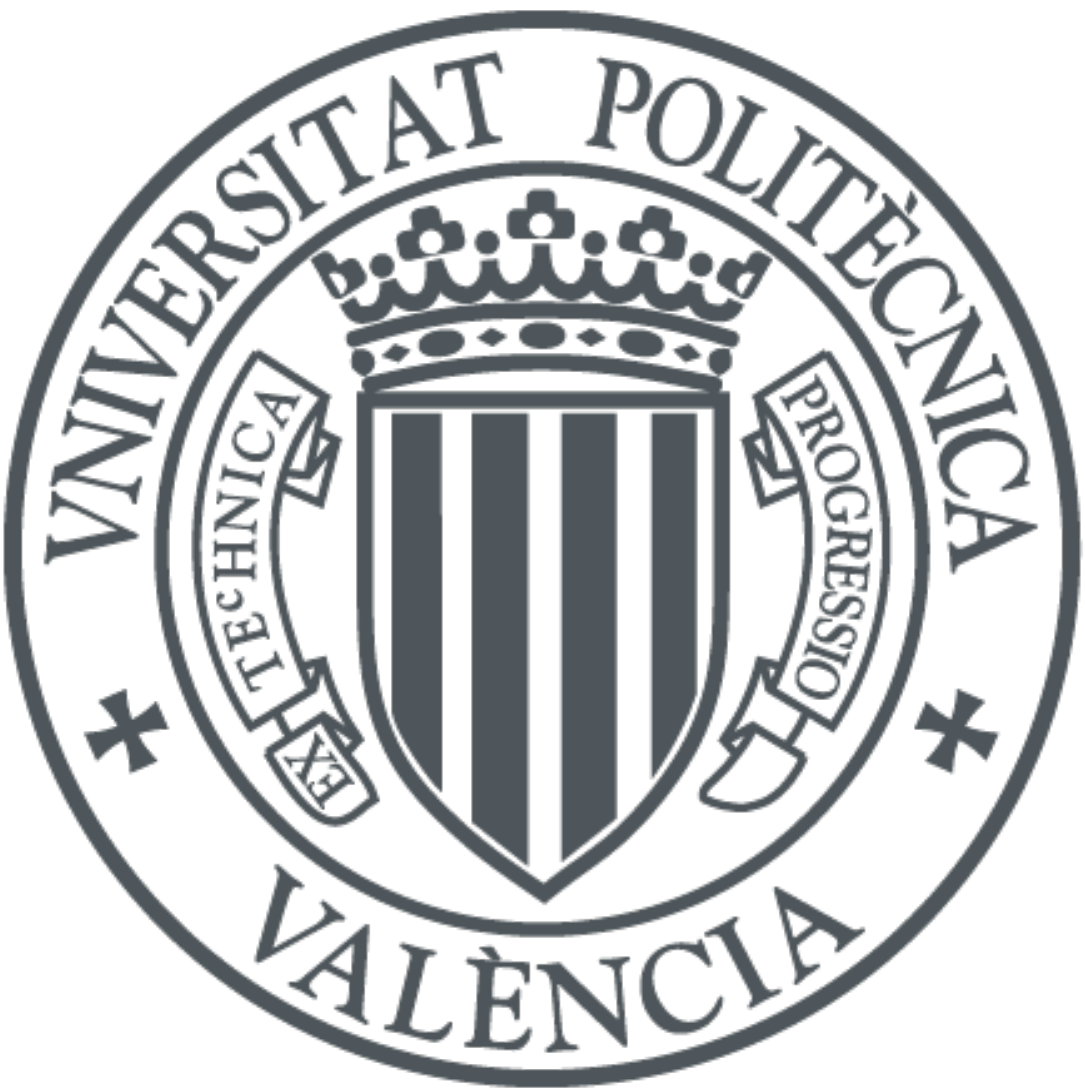

The final publication is available at

http://doi.org/10.1007/978-3-642-21796-8_16

Copyright Springer-Verlag

Additional Information 


\section{Contribution:}

Alducin G., Contero, Manuel, Martín Guitérrez J., Guerra-Zubiaga D., Johnson M.D. (2011). Productivity Improvement by Using Social-Annotations about Design Intent in CAD Modelling Process.Lecture Notes in Computer Science , 6778, 0, 153-161.

Springer International Publishing AG, Cham (hereinafter called Springer) has the exclusive, sole, permanent, world-wide, transferable, sub-licensable and unlimited right to reproduce, publish, distribute, transmit, make available or otherwise communicate to the public, translate, publicly perform, archive, store, lease or lend and sell the Contribution or parts thereof individually or together with other works in any language, in all revisions and versions (including soft cover, book club and collected editions, anthologies, advance printing, reprints or print to order, microfilm editions, audiograms and videograms), in all forms and media of expression including in electronic form (including offline and online use, push or pull technologies, use in databases and networks for display, print and storing on any and all stationary or portable end-user devices, e.g. text readers, audio, video or interactive devices, and for use in multimedia or interactive versions as well as for the display or transmission of the Contribution or parts thereof in data networks or seach engines), in whole, in part or in abridged form, in each case as now known or developed in the future, including the right to grant further time-limited or permanent rights. For the purposes of use in electronic forms, Springer may adjust the Contribution to the respective form of use and include links or otherwise combine it with other works. For the avoidance of doubt, Springer has the right to permit others to use individual illustrations and may use the Contribution for advertising purposes.

The copyright of the Contribution will be held in the name of Springer. Springer may take, either in its own name or in that of copyright holder, any necessary steps to protect these rights against infringement by third parties. It will have the copyright notice inserted into all editions of the Contribution according to the provisions of the Universal Copyright Convention (UCC) and dutifully take care of all formalities in this connection in the name of the copyright holder.

The final publication is available at Springer via:

http://dx.doi.org/10.1007/978-3-642-21796-8_16 


\title{
Productivity Improvement by Using Social-Annotations about Design Intent in CAD Modelling Process
}

\author{
Gerardo Alducin-Quintero ${ }^{1}$, Manuel Contero ${ }^{1}$, Jorge Martín-Gutiérrez ${ }^{2}$ \\ David A. Guerra-Zubiaga ${ }^{3}$, Michael D. Johnson ${ }^{4}$
}

\author{
${ }^{1}$ Universitat Politècnica de València, Camino de Vera s/n, 46022 Valencia, Spain \\ ${ }^{2}$ Universidad de La Laguna, Avda. Astrofísico Fco. Sánchez s/n, 38206 La Laguna, Spain \\ ${ }^{3}$ CIDESI Texas, Caldwell Waston Bldg., 1700 Research Pway, College Station, Texas, USA \\ ${ }^{4}$ Dept. of Engineering Technology and Industrial Distribution. Texas A\&M University, \\ 3367 TAMU, College Station, Texas, USA \\ gealqui@etsid.upv.es, mcontero@dig.upv.es, jmargu@ull.es, \\ david.guerra@cidesi.mx, mdjohnson@tamu.edu
}

\begin{abstract}
This paper focuses on the New Product Development Process (NPDP) area to contribute to increasing the productivity of CAD users by means of an improved design intent communication using a social-annotation technique. Design teams operate in a similar way to an online social network, and CAD models are not just only a 3D geometry representation, as they reflect the result of an specific modeling strategy, that usually constrains the future capability for modification and reuse of the existing 3D model. Considering this context, this work tries to assess the impact of annotations in the engineering change process in order to determine its influence in the user performance during this process. Preliminary experimental results obtained from several experiments with Spanish CAD students indicate that it is possible to reduce the time needed to perform engineering changes in existing models into a $10-20 \%$ range, if those CAD models provide annotations explaining the original design intent.
\end{abstract}

Keywords: New Product Development Process, CAD, Social-Annotations, Design Intent, Engineering Change Process

\section{Introduction}

The New Product Development (NPD) process is a key activity for the enterprise survival and competitiveness. Empowering the role of design and shortening the development cycle of new products are two well-known strategies to improve this process. In this context, advanced CAD systems are an important tool to support the NPD process, providing a key advantage to improve organizational effectiveness [1]. This paper focuses on this area in order to contribute to increasing the productivity of CAD users by enhancing design intent communication by means of a socialannotation technique, inspired on the idea that design teams operate as an online social network. 
Design intent governs the relationships between modeling features in a part, and between parts in assemblies. It is related to the behavior of the CAD system when a modification is performed on a CAD model. CAD users should analyze the geometry of the part to be generated, and plan what is the most efficient sequence of modeling features, in order to assure that future modifications in the design are managed by the CAD system in a determined expected way. Usually good CAD models are associated to change flexibility and robust variation capabilities [2] [3] [4].

Two kinds of knowledge are related to the design intent concept: declarative knowledge that is knowledge of facts (knowing that or knowing what) and procedural knowledge that is knowledge of how to do things (knowing how) [5]. This means that a CAD model is not just only a 3D geometry representation, but also this model stores the know-how about the strategy used to build it. Capture, share and transfer this complex knowledge is a key element to improve the NPD process.

Through this work is attempted to demonstrate the importance of going beyond the capture of design intent in a traditional way, based on non-explicit information associated to the feature types used in the CAD model and their interrelationship. This is aligned to Guerra-Zubiaga [6] work trying to structure different knowledge types to support manufacturing and design decisions. This work proposal includes an innovative solution by using design annotations relative to the CAD modeling strategy, specifically, about the decision making process during the 3D model construction. Design annotations give an important clue about how and why a CAD feature was used to build a CAD model by using just a few words.

This research activity is directed to assess the impact of these annotations in the engineering change process and in second place in analyzing the dynamics of the social annotation process. This paper is centered in the first aspect, presenting a pilot study that has been conducted using CAD models related to the automotive industry. These CAD models represent parts with different geometry complexity levels. For each part, two different CAD models were created, with and without annotations. These models were modified by two classes of engineering students (one mechanical engineering group at the undergraduate level, and other belonging to a master degree on $\mathrm{CAD} / \mathrm{CAM} / \mathrm{CIM}$ ). Both classes were given the same written instructions where it was explained a series of engineering changes to be performed by modifying the geometry of the CAD models. For each class a control and an experimental group were randomly organized. Experimental groups had to change the models that contained annotations about the design strategy used by the original author of the model, while the control groups had not available this additional information.

Preliminary results indicate that it is possible to reduce the time needed to perform engineering changes in existing models into a 10-20\% range by using the annotation technique. It was determined the basic knowledge-mapping and examined the problem-solving process employed by the participants in the modification of constraint-based CAD models [7]. The initial hypothesis was confirmed: the experimental group performed the design changes using less time. Additionally it was noticed that certain patterns of behavior of participants coincided with previous research works like: [1] [3] [8].

In the next point, is provided all the details about the experimental design followed to analyze the influence of annotations on the modification of CAD models. Then results are presented, explaining the limitations and main conclusions of this preliminary study and giving some orientation about future works about this topic. 


\section{Hypothesis and Methods}

\subsection{Hypothesis Definition}

This piece of research work pursues to explore how design annotations influence in the user performance during the engineering change process. This is performed before analyzing the dynamics of the social annotation process, because it is needed to assure that the availability of these annotations provides an added value to the CAD models.

This research hypothesis that CAD operators, using annotated models where original design intent is made explicit, are more efficient dealing with CAD model modifications. In this context, efficiency is related to the time used by CAD users when they have to perform a change in a $3 \mathrm{D}$ geometric model to accomplish an engineering change order.

The author's perspective is: if the design intent knowledge is made explicit by means of these annotations, the corresponding CAD models will be created with better semantic quality, following the product data quality model by Contero et al. [2]. Author's vision is that this annotation process can be performed following the behavior of social networks, where knowledge associated to the CAD modeling process is made explicit by the collaborative annotations performed by design engineers.

\subsection{Experimental Stage}

The experimental stage was divided in two phases in order to test the hypothesis: phase I, called as "undergraduate study", and phase II, named as "postgraduate study". All the participants were given the same written explanation and modeling tasks with accompanying figures, and they were asked to perform a series of engineering changes that consisted in geometric modifications on the original 3D $\mathrm{CAD}$ models. The parts were created thinking that the references used for the creation of each feature impacted the ability for later modification and edition of the geometry. This includes capturing design intent during geometry creation. Specifically, in the compound parts (e.g. automotive components) some references (e.g. datum planes or sketches) were used to separate in groups of CAD features that define each area of the part.

The used models are divided in two sets: simple geometry (low amount of CAD features used to build it) and compound geometry (composed by a high amount of CAD features). As simple geometry parts were used a housing of gear box and the part used by Johnson in [9]. As complex geometries were used a component of a PC fan housing base (from Brigham Young University's NX CAD Manual) and an automotive radiator collector (from a Spanish company). In both cases, the same type of geometry modifications was required: simple changes (e.g. modify the height of one element that is controlled by an expression) and complex changes (e.g. create a copy of a group of features with strong parametric parent-child relationships). Two variants for each $\mathrm{CAD}$ model were created: one without any annotation information explaining design intent (named regular CAD model) and other one with explicit textual annotations about the design intent (named annotated CAD model). 
The content of texts in the annotated CAD models provided information about:

- Location. This kind of text comment allows identifying the set of CAD features that define a geometric element of the part (indicating the first and last feature of a specific element).

- Design Intent. This commentary allows understanding the intention of the author of the model (e.g. this profile was used to create the main body of the connection).

- Modification Procedures. This comment gives information about both recommended steps for making modifications and procedures that must be avoided (e.g. recommendation, don't use mirror copy feature).

\subsubsection{Undergraduate Experience}

Context

Firstly, the undergraduate students group (composed by thirty students and denoted as the "undergraduate group") was split into two groups of fifteen participants. Students were enrolled in a CAD course from a Mechanical Engineering degree in La Laguna University (Spain). The course was based on Autodesk Inventor. The difference between the experimental group and control group was in the CAD model files that they received in order to conduct the study. The experimental group received CAD files with annotations, by using the Inventor Engineer Notebook functionality, that allows to create $\mathrm{CAD}$ annotations that support both text and images. The control group received the same CAD files, but stripped of any annotation information. The observed variable was the time expended in performing each modification requested to the participants. Students were given 50 minutes as maximum time to complete all the required modifications. They controlled the time, writing down the initial and final time for each requested geometric modification. Afterwards this time list was rechecked using the time stored into the participant's CAD files in order to avoid inconsistencies.

Availability of numerous groups of CAD users with a similar knowledge and expertise level in modelling is a very limited, outside the academic world. CAD students provide an interesting study population, whose behaviors can be extrapolated to the professional and industrial world. With respect to the sample size, Polkinghorne [10] and Meyer \& Booker [11] recommend a number between five and twenty designers for an exploratory phenomenological study, which is verified by the present experience. Participant students showed homogenous basic skills as Inventor users, and completed the modeling exercises as requested.

\section{Knowledge Mapping Tasks}

In both groups, participants showed a similar amount of declarative knowledge (knowledge about CAD commands) but they had a lack of procedural knowledge (knowledge of how to apply the commands to achieve a goal) due to their short experience. The high number of create-erase contiguous events was an interesting behavior pattern exhibited by the undergraduate group. This is related to the fact that they only knew the most basic commands of the CAD system, so they didn't waste time trying to use complex commands or trying to find them in the CAD user interface. The majority of the participants that completed the exercises used the most simple and direct solution. 


\subsubsection{Postgraduate Experience}

\section{Context}

The postgraduate students group (composed by eighteen students and denoted as the "postgraduate group") was divided into two groups of nine participants. Students were enrolled in an advanced CAD course from the CAD/CAM/CIM Master degree at Universitat Politècnica de València (Spain). The course was based on Siemens NX $\mathrm{CAD}$ system. The experimental group received CAD files with annotations. The control group received the same CAD files, but stripped of any annotation information. The observed variable was the time expended in performing each modification requested to the participants. Students were given 60 minutes as maximum time to complete all the required modifications. They controlled the time, writing down the initial and final time for each requested geometric modification. Afterwards this time list was rechecked using the time stored into the participant's CAD files in the same way that during the undergraduate experience.

At the beginning of this research work, was supposed that the impact of the annotations depends on several factors. One of these factors is the user knowledge and expertise. Taking into account that postgraduate students have a more extensive experience with real design problems, exercises used in this experience, have been taken from real industrial CAD models. That's the reason that in this second experimental phase was tried to organize a postgraduate students group with real design experience in the industry. This means, that they knew several CAD systems, with a medium level of expertise, being one of these systems Siemens NX. However, this group was observed to have a less homogenous behavior that the undergraduate group, due to the different background of participants.

\section{Knowledge Mapping Tasks}

It was detected that the participants' expertise influences their performance during the decision-making process to complete an engineering change request. For example if they must create a copy of a geometric element and they know that in other CAD software there is a command to perform a mirror-copy operation, they invest time to find this CAD function, although perhaps it does not exist in Siemens NX or it is not implemented in the same way than in other CAD system. This behavior is related to the fact that the annotations are easy to visualize and their content is relevant to perform the design change in a specific way. For example, if the annotations had contained direct recommendation to perform the specific design change, this expert reaction could have been avoided.

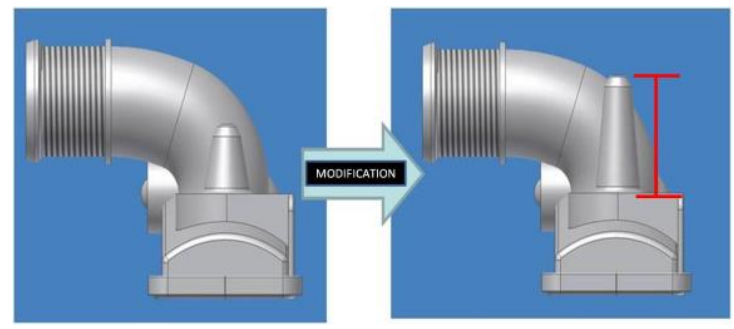

Fig. 1. Simple Engineering Change Request 


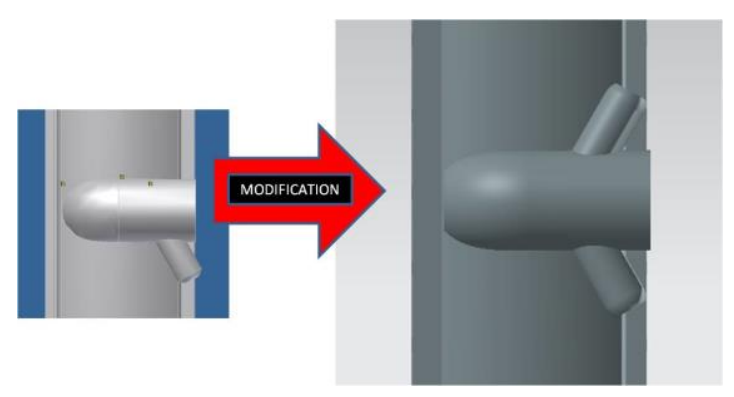

Fig. 2. Complex Engineering Change Request

\section{Results}

\subsection{General Results \& Statistical Analysis}

In the first experimental stage (undergraduate group) only fourteen students completed the engineering changes requested in exercises one and two, as it is seen in Table 1. The average time for those completing the exercise one was 37 minutes. For this exercise the experimental group (with annotations) there were six students and their average time was 28 minutes, while the control group (without annotations) there were eight students and their average time was 37 minutes. The average time for completing engineering changes in the exercise two was eight minutes. In this case the experimental group had an average of five minutes and the average time of the control group was eight minutes. In both cases the control group had an average completion time that was between 26-37\% greater than the experimental group.

During the second experimental stage (postgraduate group) the results were: for the first exercise only 16 students completed the engineering change requested with a average time of 49 minutes. Specifically the experimental group (eight students) had an average time of 43 minutes and the control group (eight students) had 52 minutes. In the case of the second exercise only 14 students completed the geometric changes requested with an average time of 50 minutes. The experimental group (seven students) had an average time of 47 minutes and for the control group (seven students) was 54 minutes. In both cases the control group had an average completion time that was between $13-17 \%$ greater than the experimental group.

Table 1. Experimental Phase I: Undergraduate Group

\begin{tabular}{|c|c|c|}
\hline Group & Control & Experimental \\
\hline Total Students Participating & 15 & 15 \\
\hline Exercise One \\
\hline Number Completing Exercise & 8 & 6 \\
\hline Average Time for Exercise Two (min.) & 37 & 28 \\
\hline Standard Deviation & 7.305 & 8.262 \\
\hline \multicolumn{2}{|c|}{ Exercise Two } \\
\hline Number Completing Exercise & 8 & 10 \\
\hline Average Time for Exercise Two (min.) & 8 & 5 \\
\hline Standard Deviation & 3.583 & 2.846 \\
\hline
\end{tabular}


Table 2. Experimental Phase II: Postgraduate Group

\begin{tabular}{|c|c|c|}
\hline Group & Control & Experimental \\
\hline Total Students Participating & 10 & 9 \\
\hline \multicolumn{2}{|c|}{ Exercise One } \\
\hline Number Completing Exercise & 8 & 8 \\
\hline Average Time for Exercise Two (min.) & 52 & 43 \\
\hline Standard Deviation & 21.715 & 20.584 \\
\hline \multicolumn{2}{|c|}{ Exercise Two } \\
\hline Number Completing Exercise & 7 & 7 \\
\hline Average Time for Exercise Four (min.) & 54 & 47 \\
\hline Standard Deviation & 17.321 & 17.153 \\
\hline
\end{tabular}

Table 3. Statistical t Analysis for Hypothesis

\begin{tabular}{|c|c|c|c|c|}
\hline \multirow{2}{*}{$\begin{array}{c}\text { Exercise } \\
\text { / Phase }\end{array}$} & \multicolumn{2}{|c|}{ Time (minutes) } & \multirow{2}{*}{$\mathrm{T}$} & \multirow{2}{*}{ Significance } \\
\cline { 2 - 3 } & Control & Experimental & & \\
\hline $\mathrm{E} 1 / \mathrm{Ph} \mathrm{I}$ & 37 & 28 & -2.243 & 0.045 \\
\hline $\mathrm{E} 2 / \mathrm{Ph} \mathrm{I}$ & 8 & 5 & -2.165 & 0.046 \\
\hline $\mathrm{E} 1 / \mathrm{Ph} \mathrm{II}$ & 54 & 43 & -0.993 & 0.338 \\
\hline $\mathrm{E} 2 / \mathrm{Ph} \mathrm{II}$ & 54 & 47 & -0.729 & 0.480 \\
\hline
\end{tabular}

While the results in the first phase (undergraduate group) showed that there is difference between control and experimental groups, the results were further analyzed to detect any statistically significant differences. The results of those statistical tests are shown in the table 3 . This table shows the relevant statistic and one-tailed probability. Although in all the cases, experimental group (who's received additional information through annotations) showed a better performance, this means they used less time than the control group. But only there is a statistically significant difference with the undergraduate group experience. The authors relied on that is because the undergraduate group has more homogenous understanding about design and CAD software than other groups [12].

\section{$4 \quad$ Limitations and Future Work}

One of the most important limitations of this research work relates to the sample size of the CAD users that participated in the experiences. The sample size was small as this pilot study, being exploratory in nature, was intended to give us suggestions for more extensive studies in the future, where larger number of participants would allow to perform a more robust statistical analysis. Other factor that can be considered a limitation is the users' expertise. The undergraduate group students showed low level CAD skills (they were enrolled in a basic level CAD course) but a homogenous background. This was a positive factor to obtain a result concordant with the initial hypothesis. The postgraduate group of students had a different profile. The majority of them had a real industry experience in design, showing a heterogeneous professional background with different CAD skills. This heterogeneity could have let to the lack of more conclusive results. 
Future work will be focused on mitigating some of these limitations mentioned above and improving the analysis of the results. The first step would be to find a larger group of students for participating in the experiments. They should offer a similar experience and expertise. Second step would be the redesign of experiments applying all the experience obtained during the previous study.

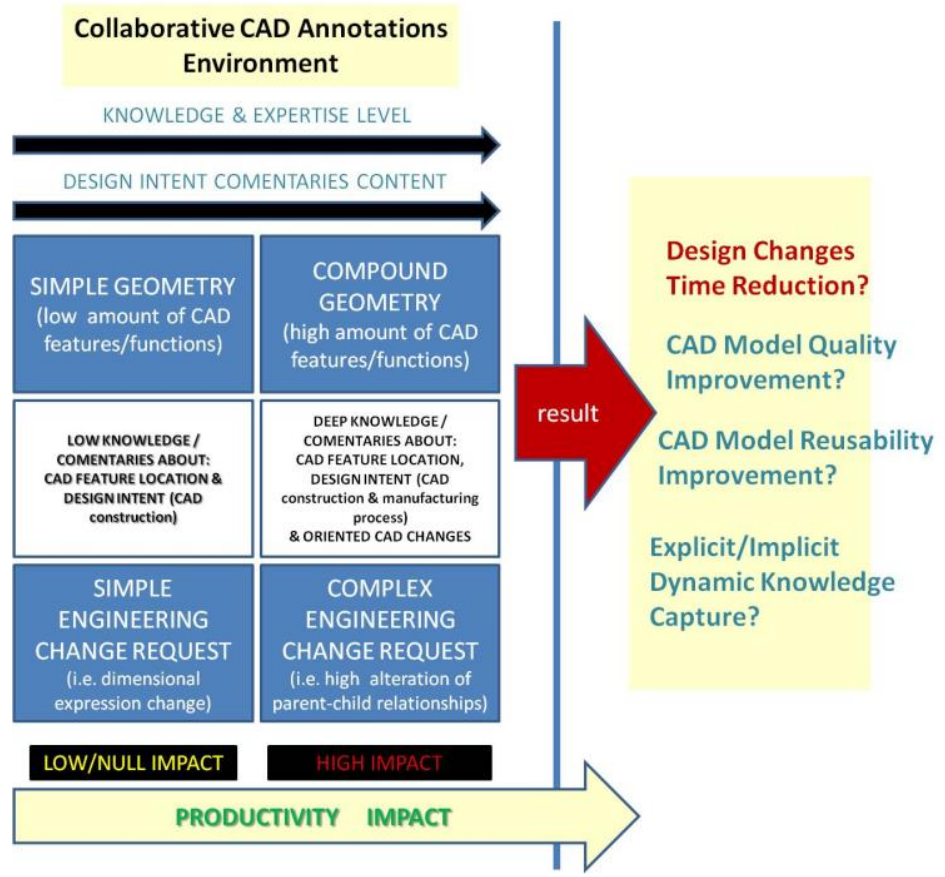

Fig. 3. Impact of Design Intent Annotations

\section{Conclusions}

Product design can be considered a social process involving groups of individuals (i.e., design teams) attempting to meet complex product needs through sharing varied expertise and knowledge types [13]. It is a creative process involving networks of people with negotiations and interactions between them; the product design itself is dynamic and evolving $[14,15]$. This work explored the effects that design intent annotations had during the engineering change process and assessed the possible relationship between annotations' content and the designer's knowledge related to an expertise level.

The findings presented in this paper show that the productivity impact through CAD annotations depends of several factors such as geometry among others. It can be shown that if a simple design change is performed on a simple geometry the annotation impact is low or null. However, if a complex change is required on complex geometry the annotation impact is significant. In other words, the annotation impact depends on how it can be structures according to the annotation's content and the user's knowledge as shown in figure 3. 
It has been shown the need of explore new knowledge infrastructure to support better design decisions, not depending on a particular computer design platform through product design development.

\section{Acknowledgement}

The authors acknowledge the support received by UPV, CIDESI-TEXAS, Texas A\&M University, and Universidad de la Laguna. The authors also acknowledge the support by Tecnológico de Monterrey through the Research Chair in Nanotechnology and the valuable support of Oscar Martínez and Germán Manacera on the experimental performance of this piece of work.

\section{$7 \quad$ References}

1. Anderl, R., \& Mendgen, R.: Parametric design and its impact on solid modeling applications. In: Third ACM Symposium on Solid Modelling and Applications, ACM, pp. 1--12 (1995).

2. Vila, C., Contero, M., \& Company, P.: Extended modeling, a tool for cooperative design. In: 6th International Conference on Concurrent Enterprising, Tolouse France $(2000)^{*}$

3. Anderl, R., Mendgen, R.: Analyzing and optimizing constraint-structures in complex parametric CAD models, in: B. Bruderlin, D. Roller (eds.), Geometric Constraint Solving and Applications, Springer, Berlin, Germany, pp. 58--81 (1998).

4. Hartman, N. W.: The development of expertise in the use of constraint-based CAD tools. Eng. Design Graph. J. 68, 14--26 (2004)

5. Rynne, A.: AC 2007-2132: Cognitive modelling strategies for optimum design intent in parametric modelling (PM). American Society for Engineering Education (2007).

6. Guerra-Zubiaga, D.A., A Model to enable Knowledge Maintenance: Supporting Manufacturing Decisions, publisher: VDM Verlag, Germany. ISBN 9783639209983, (2009)

7. Hartman, N. W.: Defining expertise in the use of constraint-based CAD tools by examining practicing professionals. Eng. Design Graph. J. 68, 6--15 (2005)

8. Bhavnani, S. K., Garrett, J. H., J., \& Shaw, D. S.: Leading indicators of CAD experience: Paper presented at the Proceedings of Computer-Aided Architectural Design Futures CAAD Futures'93, pp. 313-34 (1993)

9. Johnson, M.D., Diwakaran, R. P.: Assessing the effect of incentive on computeraided design intent. In: the ASME 2009 International Design Engineering Technical Conferences \& Computers and Information in Engineering Conference, pp. 523-532. San Diego, California (2009)

10. Polkinghorne, D.E.: Phenomenological research methods. In: R.S. Valle \& S. Halling (eds.) Existential-phenomenological perspectives in psychology, pp. 41--60. New York Plenum (1989)

11. Meyer, M.A. \& Booker, J.M.: Eliciting and analyzing expert judgment: A practical guide. Academic Press, San Diego, CA (1991)

12. Reddy, J. M., Finger, S., Konda, S., Subrahmanian, E.: Design as Building and Reusing Artifact Theories: Understanding and Supporting Growth of Design Knowledge. In: The Design Productivity Debate, Springer (1998)

13. Bucciarelli, L. L. Designing engineers. MIT Press, Cambridge MA (1994) 
14. May, P., Ehrlich, H.C., Steinke, T.: Mapping Sociotechnical Networks in the Making. In: Beyond the Great Divide, Bowker, G. Star, S. L., Turner, W., Gasser, L. (eds.) Technical Systems and Co-operative Work. Lawrence Erlbaum (1997).

15. Bhavnani, S.K., Garrett, J.H.: Leading Indicators of CAD Experience, in: U. Flemming and S. Van Wyk (eds.), CAAD Futures '93, Elsevier Science Publishers, Netherlands, pp. 313--334 (1993). 